\title{
Extreme Thrombocytosis not always Essential Thrombocythemia- BCR-ABL has to be Done Timely
}

\author{
Uzma Mahar, Nida Anwar*, Jawad Hassan, Tahir Sultan Shamsi \\ Department of Hematology, National Institute of Blood Disease \& Bone Marrow Transplantation, Karachi, Pakistan.
}

\begin{abstract}
Chronic Myeloid Leukemia (CML) is a myeloproliferative neoplasm in which the major proliferative component is comprised of granulocytes and is characterized by translocation of BCR-ABL and formation of Philadelphia (Ph) chromosome. Extreme thrombocytosis in chronic myeloid leukemia is rare and mimic essential thrombocythemia. Thus, chronic myeloid leukemia should be ruled out in patients who present with extreme thromobocytosis. A 28 years old female presented to us with abdominal discomfort, bleeding gums and hematuria. Her past medical history was unremarkable and there wasn't any family history of bleeding disorder. On examination she had pallor and splenomegaly. Laboratory studies showed thrombocytosis with platelet count of $6420 \times 10^{\wedge} 9 / \mathrm{L}$, moderate leucocytosis of $22.8 \times 10^{\wedge} 9 / \mathrm{L}$ along with low hemoglobin of $7.9 \mathrm{~g} / \mathrm{dL}$. Workup for acquired von willebrand disease was done which showed vWF: Ag 94\%, vWF: Ricof 30\%, FVIII $75 \%$. Her cyto reduction with hydroxycarbamide $3 \mathrm{~g}$ /day was started and patient was proceeded for platelet pheresis. On follow up after two sessions of platelet pheresis her platelet count was still high $3104 \times 10^{\wedge}$ 9/L. JAK2V617F, MPL, CALR and BCR-ABL mutations were checked and she turned out to be BCR-ABL positive, rest of the mutations were not detected. Bone marrow biopsy findings were consistent with chronic myeloid leukemia in accelerated phase along with marked proliferation of megakaryocytes present in sheets. Anagrelide was administered to control thrombocytosis and nilotinib $800 \mathrm{mg}$ /day was started. Although chronic myeloid leukemia can present with thrombocytosis but platelet count exceeding $1000 \times 10^{\wedge} 9 / \mathrm{L}$ is rare. Our patient demonstrated very high count of platelets $\left(6420\right.$ x $\left.10^{\wedge} 9 / \mathrm{L}\right)$ and relatively unremarkable white cell count. Most of the symptoms she had were due to thrombocytosis rather than leucocytosis as typically seen in chronic myeloid leukemia.
\end{abstract}

Keywords: Extreme thrombocytosis, Essential thrombocythemia, BCR-ABL, Timely evaluation, Chronic myeloid leukemia (CML), Hematuria.

\section{INTRODUCTION}

Chronic Myeloid Leukemia (CML) is a myeloproliferative neoplasm in which the major proliferative component is comprised of granulocytes and is characterized by translocation of BCR-ABL and formation of Philadelphia $(\mathrm{Ph})$ chromosome [1]. There are three phases of chronic myeloid leukemia, an initial chronic phase which is followed by an accelerated phase or a blast phase. Majority of patients are diagnosed in chronic phase and among those half of the patients are asymptomatic. Symptomatic patients usually present with fatigue, malaise, weight loss, night sweats and splenomegaly [2]. Most of the cases can be diagnosed on the basis of peripheral blood findings and detection of Philadelphia chromosome by cytogenetics or BCR-ABL by molecular genetic techniques [1]. However, bone marrow biopsy is important to confirm the phase of the disease. Extreme thrombocytosis in chronic myeloid leukemia is rare and mimic essential thrombocythemia. So chronic myeloid leukemia should also be ruled out in patients who present with extreme thromobocytosis [2].

\section{CASE REPORT}

Twenty eight years old female presented with complaint of

*Address correspondence to this author at the Department of Hematology, National Institute of Blood Disease \& Bone Marrow Transplantation,

Karachi, Pakistan. E-mail: drnidairfan@yahoo.com abdominal pain for 01 month, gum bleeding and hematuria for 03 days. Her past medical history was unremarkable and there wasn't any family history of bleeding disorder. Her previous medical record revealed $\mathrm{CBC}$ in which her platelet count was $4767 \times 10^{\wedge} 9 / \mathrm{L}$, total leucocyte count (TLC): $26.4 \times 10^{\wedge} 9 / \mathrm{L}$ and hemoglobin $(\mathrm{Hb}): 8.0 \mathrm{~g} / \mathrm{dL}$. On examination there was pallor and splenomegaly. Laboratory studies showed thrombocytosis with platelet count of $6420 \times 10^{\wedge} 9 / \mathrm{L}$ and moderate leucocytosis of $22.8 \times 10^{\wedge} 9 / \mathrm{L}$ along with low hemoglobin of $7.9 \mathrm{~g} / \mathrm{dL}$. On further evaluation, her ultrasound abdomen showed mild hepatosplenomegaly and there wasn't any evidence of thrombosis. Keeping in view the history of hematuria, gum bleeding and persistently raised platelet count, her workup for acquired von willebrand disease was done which showed vWF: Ag 94\%, vWF: Ricof 30\%, FVIII $75 \%$. Her cyto reduction with hydroxycarbamide $3 \mathrm{~g} /$ day was started and patient was proceeded for platelet pheresis. On follow up after two sessions of platelet pheresis her platelet count was still high $3104 \times 10^{\wedge} 9 / \mathrm{L}$. Her JAK2 V617F, MPL, CALR and BCR-ABL mutations were checked and she turned out to be BCR-ABL positive, rest of the mutations were not detected. Bone marrow biopsy findings were consistent with chronic myeloid leukemia in accelerated phase and showed marked proliferation of megakaryocytes present in sheets (Figs. 1 \& 2). Two weeks later she presented with right sided leg pain. Despite the high dose of hydroxyurea her platelet

www.njhsciences.com 
count raised to $4143 \times 10^{\wedge} 9 / \mathrm{L}$. At this point, further two sessions of platelet pheresis were carried out and anagrelide was administered to control thrombocytosis and nilotinib $800 \mathrm{mg} /$ day was started.

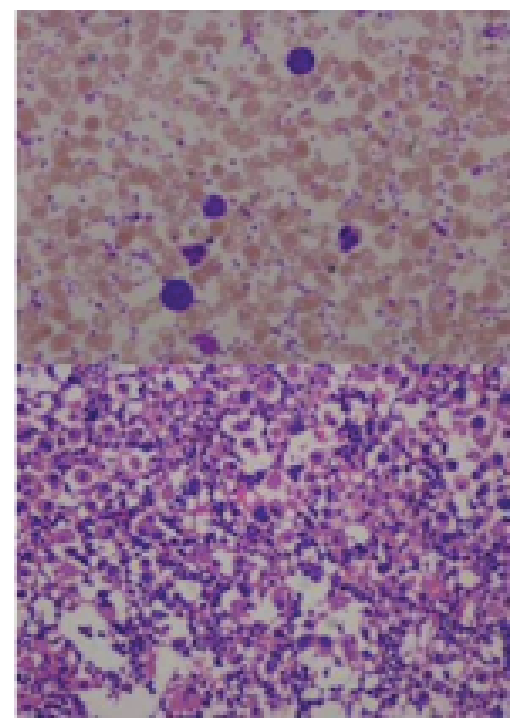

Fig. (1). Peripheral Blood Film and Bone Marrow Trephine.

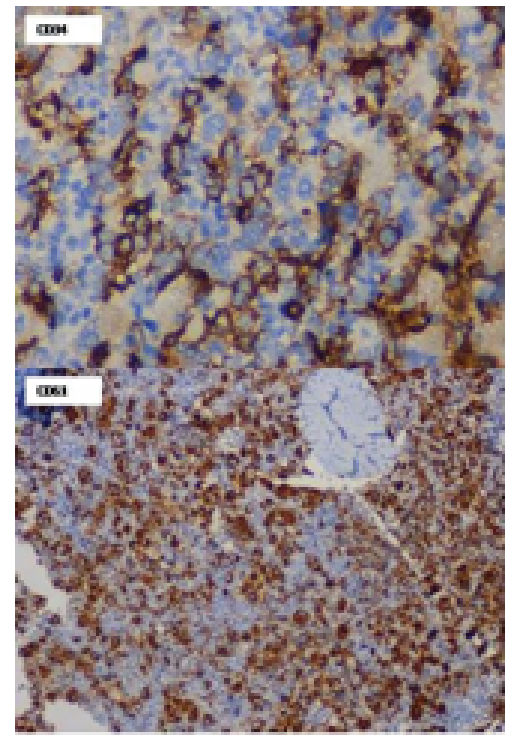

Fig. (2). CD 34 and CD 61 on Immunohistochemistry.

On examination there was tenderness along with some degree of swelling and we suspected thrombosis so doppler ultrasound was done which showed small thrombus in right common femoral vein.

However, anticogulation was not started as the patient refused to receive anticoagulants and was discharged. On follow up after one week, her platelet count was within normal range. Thus, anagrelide and hydroxyurea were stopped and patient was continued on nilotinib alone.
Although chronic myeloid leukemia can present with thrombocytosis but platelet count exceeding $1000 \times 10^{\wedge} 9 / \mathrm{L}$ is rare [3]. Our patient demonstrated a very high count of platelets $\left(6420 \times 10^{\wedge} 9 / \mathrm{L}\right)$ and relatively unremarkable white cell count. Most of the symptoms she had were due to thrombocytosis rather than leucocytosis as typically seen in chronic myeloid leukemia. Additionally, our patient was in accelerated phase on bone marrow biopsy.

CML is caused by translocation of breakpoint cluster region protien (BCR) gene to Ableson murine leukemia viral oncogene homolog 1 (ABL), which results in the production of unregulated tyrosine kinase and leads to cell proliferation without being regulated by cytokines [4]. At the genetic level, four different breakpoints have been identified in BCR and the most common breakpoints involve the M-bcr region comprised of BCR interons downstream of either exon 13 (e13 or b2) or 14 (e14 or b3) and interons upstream of $\mathrm{ABL}$ exon 2 (a2) [5]. In patients who present with extreme thrombocytosis, e14a2 breakpoint subtype has been identified [3]. However, cases with atypical transcript such as e14a3 have also been reported [5].

A study has suggested that chronic myeloid leukemia with extreme thrombocytosis is more common in female with intermediate to high risk according to sokal score. However, the cytogenetic and molecular responses of these patients were not significantly different from the patients without extreme thrombocytosis [3].

It has also been observed that this subset of patients do not respond to hydroxycarbamide cytoreduction and the only way to control their platelet count is to start TKI-therapy, as seen in our patient $[2,3]$. Mechanical removal of platelets by aphresis can be considered in patients who develop thrombo-hemorrhagic complications of thrombocytosis [6].

Risk of thrombo-hemorrhagic complications associated with thrombocytosis were reported to be insignificant and less severe in literature $[3,7]$. On contrary, our patient did present with this complication, which responded well when her platelet count was decreased to normal range.

\section{CONCLUSION}

In conclusion, on one hand, essential thrombocythemia follows an indolent course and on the other hand, chances of chronic myeloid leukemia transformation to blast crises are more, if left untreated. So patients who present with extreme thrombocytosis should be evaluated for CML because timely diagnosis and initiation of TKI-therapy has a crucial role in management of such patients.

\section{LIST OF ABBREVIATIONS}

Chronic myeloid leukemia (CML)

Philadelphia $(\mathrm{Ph})$ 
Breakpoint Cluster Region Protien Gene (BCR)

Ableson Murine Leukemia Viral Oncogene Homolog 1 (ABL)

\section{CONFLICT OF INTEREST}

Declared none.

\section{ACKNOWLEDGEMENTS}

Declared none.

\section{REFERENCES}

[1] Frazer R, Irvine AE, McMullin MF. Chronic myeloid leukaemia in the 21 st century. Ulster Med J 2007; 76(1): 8.

[2] Byun YJ, Park BB, Lee ES, Choi KS, Lee DS. A case of chronic myeloid leukemia with features of essential thrombocythemia in peripheral blood and bone marrow. Blood Res 2014; 49(2): 127-9. DOI: 10.5045/br.2014.49.2.127

[3] Sora F, Iurlo A, Sica S, et al. Chronic myeloid leukaemia with extreme thrombocytosis at presentation: Incidence, clinical findings and outcome. BJH 2018; 181(2): 267.

DOI: $10.1111 /$ bjh. 14553

[4] Ng TF, Wright M, De Kraa R. Approximately 1\% of chronic myeloid leukaemia cases present with isolated thrombocytosis and express common major breakpoints: A finding from a laboratory audit. Pathology 2019; 51(1): 98-9.

DOI: 10.1016/j.pathol.2018.09.059

[5] Chisti MM, Sanders DS. Chronic myeloid leukemia with b3a3 (e14a3) fusion: A rare BCR/ABL rearrangement presenting with thrombocytosis-does MTHFR polymorphism matter. Case Rep Oncol 2018; 11: 485-92. DOI: 10.1159/000490697

[6] Ebrahem R, Ahmed B, Kadhem S, Truong Q. Chronic myeloid leukemia: A case of extreme thrombocytosis causing syncope and myocardial infarction. Cureus 2016; 8(2): e476.

DOI: $10.7759 /$ cureus.476

[7] Liu Z, Fan H, Li Y, Liu C. Analysis of clinical characteristics and efficacy of chronic myeloid leukemia onset with extreme thrombocytosis in the era of tyrosine kinase inhibitors. Onco Targets Ther 2017; 10: 3515. DOI: 10.2147/OTT.S142587 